\title{
Modelado y simulación de procesos de laminado conjunto por límite superior
}

\author{
H.A. Quiroz-González, A. Ortiz-Prado, Y. Houbaert-Irmen \\ Departamento de Ingeniería M ecánica, Facultad de Ingeniería, UNAM \\ E-mails: adrián_quiroz@correo.unam.mx,armandoo@ servidor.unam.mx,yvan.Houbaert@ rug.ac.be
}

(recibido: agosto de 2002; aceptado: septiembre de 2003)

\begin{abstract}
Resumen
El presente artículo muestra un modelo para la simulación de procesos de laminado conjunto basado en un análisis por límite supe rior y el uso de una función de corriente para los flujos de mate rial de las capas que se colaminan. El modelo predice el comportamiento de la deformación plástica de las capas en el claro de laminación. Los campos de velocidad se derivan a partir de funciones de corriente que garantizan el cumplimiento de condiciones de frontera, así como la conservación del volumen. Se estudia el efecto de la variación de condiciones de laminado, tales como el factor de fricción entre el mate rial de la capa externa, el rodillo, la reducción y la relación de espesores al inicio del proceso.
\end{abstract}

Descriptores: deformación plástica, límite supe rior, laminación, colaminación, conformado mecánico.

\begin{abstract}
This pa per in tro ducean up per bound so lu tion for simetric clad ding, it uses Stream Lines for flow description. The proposed model predicts theplas tic defor ma tion behaviour of theup per and in ner ma terial flow and could beusefor thecal cu la tion of theout thick ness ra tio. The ve loc ity fields are descripted with the use of thestream linefunctions wich war ranty the pro ces $s$ boundary con ditions and the vol ume con ser va tion. This model could beus ing for the ef fect anal y sis of theroll ing vari ables likethefriction between thebound aries (roll-upper ma terial, up per-inner ma terial), mechan i cal propierties (yield stress, work hard en ing co ef fi cient) and thein ner thick ness ratio.
\end{abstract}

Keywords: plasticdefor ma tion, up per bound theory, roll ing, clad ding, met al working process.

\section{Introducción}

La diferencia entre el estado de esfuerzos de las capas que se colaminan du rante los procesos de laminado conjunto, en la mayoría de los casos, conduce a una variación notable en los espesores relativos al final del proceso, así los mecanismos de deformación plástica en el claro de laminación resultan más complejos que en el caso de laminación 
convencional y con ello el análisis del proceso. Aún cuando existen trabajos publicados con relación a este proceso desde la década de 1970, en su mayoría fueron basados en la experimentación, por lo tanto, su alcance en cuanto a generalidad se refiere, resulta ser muy limitado.

Los pocos análisis teóricos desarrollados en aquella época resaltaban por la complejidad del cálculo numérico necesario, lo que se traducía en tiempo excesivo de procesador y cuya precisión dejaba que desear en relación con los resultados experimentales.

Estudios recientes han avanzado en el planteamiento matemático del problema, principalmente en la década de 1990, con lo que se facilita obtener un conocimiento más profundo de lo que ocurre en los procesos de colaminado, esto gracias a la creciente aceptación de los métodos denominados de límite, los cuales incluso compiten en ciertas aplicaciones con métodos de solución más complejos como puede ser el caso del Método del Elemento Finito (MEF). Los estudios más relevantes relativos a los procesos de colaminado desprecian el efecto del deslizamiento en la interfase entre los materiales que se colaminan. En este artículo se han retomado los conceptos fundamentales planteados por Hwang (1995); sin embargo, el efecto del deslizamiento entre las capas se ha incluido en el modelo desarrollado y se demuestra su importancia para efecto de determinar los cam pos de velocidades de cada uno de los flujos de material. Adicionalmente, se hace una evaluación de la evolución de los pseudo-parámetros durante el proceso de optimación, lo que conducirá a caracterizar su comportamiento y estabilidad durante el proceso.

\section{Teorema de límite superior}

Del principio de Stokes se deriva el teorema de límite superior, el cual indica que si se desprecia el cambio en la energía cinética del cuerpo, entonces se debe cumplir lo siguiente:

$$
\dot{w}_{n} * \leq \dot{w}_{i} *
$$

en donde $\dot{w}_{n}$ es la potencia neta desarrollada por las fuerzas externas y $\dot{\mathrm{w}}_{i}$ es la potencia interna consumida por la deformación.

El campo de velocidad $v^{*}$ a partir del cual se obtienen ambos términos de la ecuación (1) puede ser seleccionado arbitrariamente, la condición más estricta que debe cumplir es la de incompresibilidad. Así, los campos de velocidades que cumplen lo anterior se denominan cinemáticamente admisibles. EI campo de velocidades que minimice la desigualdad expresada en la ecuación (1) es el más próximo al campo de velocidades real del flujo de ma te rial.

Una representación más elaborada y quizá más adecuada de dicho teorema está dada por el Principio Minimum de Markov, el cual se basa en los principios del cálculo de variaciones y se ajusta a campos de velocidades discontinuos. Este principio establece lo siguiente:

"De todos lo campos admisibles de velocidad, el único que minimiza la funcional J* es el campo de velocidad del flujo real".

De forma que:

$$
\dot{\mathrm{W}}_{\mathrm{v}}=\mathrm{J} \leq \mathrm{J}^{*}
$$

Donde $\dot{W}_{v}$ es igual a la potencia neta desarrollada por todas las fuerzas externas 
que son aplicadas con una velocidad conocida.

Del principio de Markov se concluye que el mínimo valor J, de la funcional J*, proporciona el campo de velocidad que asegura la potencia necesaria para mantener el flujo plástico del material.

Usualmente, el valor mínimo de J es desconocido y no puede ser obtenido. En vez de ello, se calcula J* para un conjunto de campos de velocidades seleccionados y se elige el de valor más bajo al cual J* conduce. De esta manera, el problema deriva en un proceso de optimación, en donde el campo de velocidades que minimice J* será la solución óptima.

\section{Procesos de colaminado. Modelado geométrico y cinemático. Análisis por LS}

El planteamiento de una solución por límite superior para procesos de colaminado, requiere que las siguientes condiciones sean satisfechas:

1. Se desprecia toda deformación de los rodillos.

2. Las deformaciones en los materiales que se colaminan son consideradas como biaxiales.

3. Los materiales son considerados rígido-plásticos.

4. La fricción entre la capa superior y el rodillo, así como la existente entre las mismas capas se considera que obedece al modelo de fricción constante, es decir, que el esfuerzo cortante es proporcional a la resistencia de los materiales.

5. Los campos de velocidades de ambos materiales se unifican a la salida, con lo cual se obtiene un solo perfil de velocidades, lo que indica que los materiales se han unido de manera permanente.

\section{Análisis del flujo de material en la capa superior}

El campo de velocidades para la región que comprende el claro de laminación que cumple con condiciones de frontera para el caso en estudio, se deriva a partir de la siguiente función de corriente:

$$
\phi_{1}=Q_{1}\left\{\frac{y-y_{2}}{y_{1}-y_{2}}+C_{1}\left(y-y_{2}\right)\left(y-y_{1}\right)\right\}
$$

Donde:

$Q_{1}$ : Gasto volumétrico de la capa superior.

$Y_{1}$ : Frontera superior. (Superficie del rodillo).

$\mathrm{Y}_{2}$ : $\quad$ Frontera inferior. (Interfase de los materiales).

$C_{1}$ : Gradiente de perfil de velocidad linealmente distribuido.

El primer término dentro de las llaves corresponde a un flujo uniforme, el cual es escalado en función de la geometría de las fronteras que restringen al flujo. El segundo término corresponde a un flujo linealmente distribuido, que de igual manera, es escalado en función de las fronteras del flujo. La forma del flujo quedará definida entonces en términos de la posición relativa de una partícula respecto a las fronteras.

La figura 1 muestra el esquema del proceso y la nomenclatura empleada para el desarrollo del modelo. 


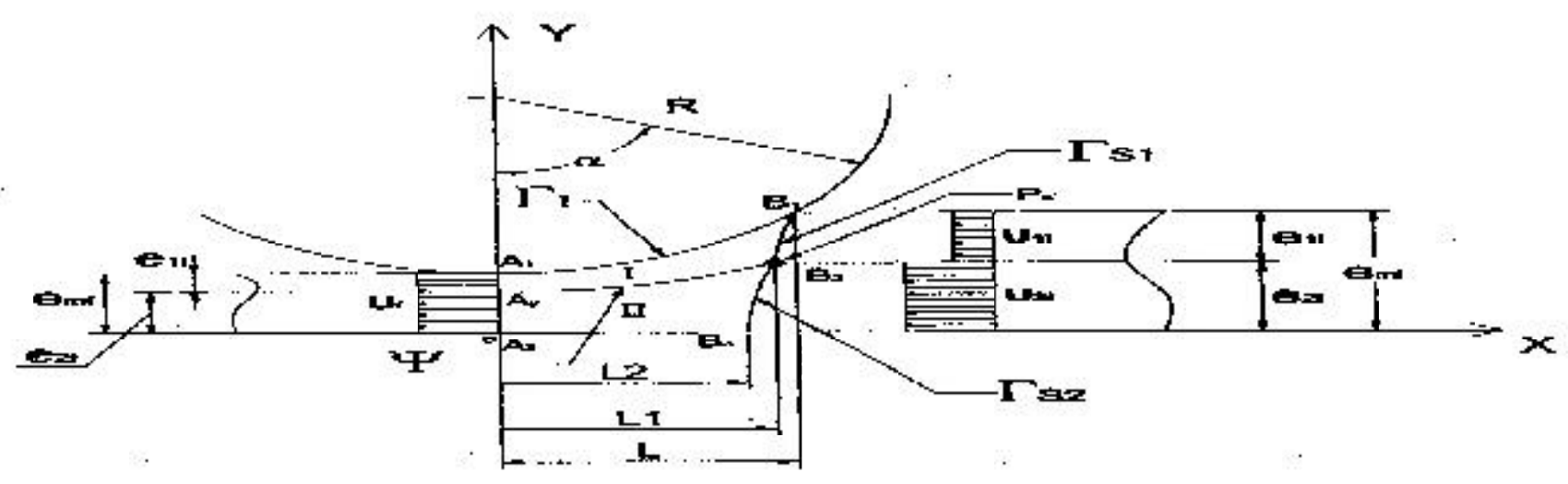

Figura 1

El término que representa el flujo linealmente distribuido en sí, muestra en gran medida una variación del flujo uniforme que, en caso de una reducción de magnitud cero, se presentaría; el término $C_{1}$ es un escalamiento de dicha variación y puede considerarse como un gradiente de la distribución lineal de velocidad. Considerando la forma de las herramientas (en este caso rodillos), la velocidad varia en función de la coordenada $x$, es lógico asumir que $C_{1}$ puede ser función de $x$ (y en realidad de y pero por simplicidad del modelo se considera función exclusiva de $x$ ). De forma que:

$$
C_{1}=f(x)
$$

Se puede definir el primer término dentro de las llaves de la siguiente manera:

$$
\eta=\frac{y-y_{2}}{y_{1}-y_{2}}
$$

Considerando flujo en la capa superior, las fronteras quedan definidas de la siguiente manera:

$$
y_{1}(x)=R+e_{m f}-\sqrt{R^{2}-x^{2}}
$$

(Representa la superficie del rodillo).

$$
y_{2}(x)=e_{2 f}+b x^{2}
$$

(Representa la interfase de los materiales).

El gasto volumétrico ${ }^{1}$ en dicha capa es $Q_{1}$ y el gradiente $C_{1}$, para el flujo de la capa su pe rior se toma como una relación cuadrática de la coordenada en $x$.

$$
c_{1}(x)=a_{1} x^{2}
$$

El parámetro $a_{1}$ es un parámetro pseudoindependiente que será optimado y representa la amplitud del comportamiento parabólico del gradiente en la dirección $x$ del perfil linealmente distribuido para la capa superior.

La frontera in fe rior del flujo de la capa su perior es la interfase de los materiales, cuyo perfil es deducido considerando una posible forma primitiva de la misma (sin considerar las deformaciones localizadas que podrían hacer de ésta una superficie más compleja, en donde la representación analítica sería al mismo tiempo más complicada). Con ello, no es difícil

\footnotetext{
1 Dado que se consideran deformaciones planas, la potencia y la fuerza quedan expresadas por unidad de ancho.
} 
suponer que una forma cuadrática se adaptará bien a la forma de la interfase. Así, a través del valor del parámetro pseudo-independiente $b$, se convierte en parte de la solución del problema de optimación a re solver.

Una vez definida plenamente la función de corriente, las componentes del campo de velocidades se obtienen de manera sencilla:

$$
\begin{array}{r}
U_{1}=\frac{\partial \phi}{\partial y}=Q_{1}\left\{\frac{1}{y_{1}-y_{2}}+2 C_{1}\left[y-\frac{\left(y_{1}+y_{2}\right.}{2}\right]\right\} \\
V_{1}=-\frac{\partial \phi}{\partial x}=Q_{1}\left\{\eta^{\prime}+C^{\prime}{ }_{1}\left(y-y_{1}\right)\left(y-y_{2}\right)-\right. \\
\left.C_{1}\left(y-y_{2}\right) y^{\prime}{ }_{1}-C_{1}\left(y-y_{1}\right) y^{\prime}{ }_{1}\right\}
\end{array}
$$

donde:

$$
\eta^{\prime}=\frac{\left(y_{1}-y_{2}\right)\left(-y_{2}^{\prime}\right)-\left(y-y_{2}\right)\left(y^{\prime}{ }_{1}-y_{2}^{\prime}\right)}{\left(y_{1}-y_{2}\right)^{2}}
$$

y donde la prima denota la derivada respecto ax.

\section{Superficies de discontinuidad de velocidad2}

Debido a que el perfil de velocidades resultante sólo es válido para la región que comprende el claro de laminación, es evidente que existirá una discontinuidad de velocidad entre el flujo de la zona previa al claro de laminación y el flujo dentro de éste. Estas discontinuidades se conocen comúnmente como superficies de discontinuidad de velocidad y en el caso partic ut lar que se estudia, se les puede considerar como límites rígido-plásticos, puesto que es a partir de ellos y en la dirección del flujo que el material comienza a deformarse plásticamente por los rodillos, y aún cuando su existencia se basa exclusivamente en la necesidad intrínseca de describir un campo de velocidades por medio de superposición sucesiva de campos de velocidades, lo que facilita la representación del campo real, se ha comprobado experimentalmente (Avitzur, 1980), que no sólo se justifica su existencia, sino que ésta resulta ser una realidad en los procesos de deformación plástica. Aquí por ejemplo, parte del material puede quedar adherido a las herramientas, generándose así discontinuidades en el campo de velocidades.

La función que de scribe el perfil de velocidades en la región previa al campo de deformación es aquella que finalmente conduce a un perfil uniforme de velocidad, por lo que definiendo a $\phi_{2}$ como la función de corriente para el flujo en la región previa al claro de laminación:

$$
\phi_{2}=Q_{1}\left\{\frac{y-e_{2 i}}{e_{1 i}}\right\}
$$

Debido a la continuidad de las líneas de corriente $\left(\phi_{1}=\phi_{2}\right)$ en la entrada del claro de laminación, la función que describe la forma del límite rígido-plástico $y_{3}$ a la entrada del claro de laminación $\Gamma_{\text {sl }}$ es:

(13)

$$
y_{3}=y_{3}(x)=\frac{-K+\sqrt{K^{2}-4 C_{1} M}}{2 C_{1}}
$$

$$
\begin{gathered}
K=\frac{1}{y_{1}-y_{2}}-C_{1}\left(y_{1}+y_{2}\right)-\frac{1}{e_{1 i}} \\
D=C_{1} y_{1} y_{2}-\frac{y_{2}}{y_{1}-y_{2}}+\frac{e_{2 i}}{e_{1 i}}
\end{gathered}
$$

La ecuación (14) es la solución de una ecuación de segundo orden que resulta de

2 Conocidas también como Superficies de Corte, o bien, Límites Rígido-Plásticos. 
igualar las funciones de corriente correspondientes a cada una de las regiones, por lo tanto, existen aparentemente dos soluciones que satisfacen la continuidad en las líneas de corriente. Sin embargo, al elaborar las representaciones gráficas de dichas soluciones es evidente el hecho de que aquella solución con el signo positivo en el radical involucrado es la representación adecuada de dicho límite.

\section{Análisis del flujo de material en la capa inferior}

La capa inferior, a diferencia de la superior, tiene como límites la propia interfase de ambos materiales y el plano medio 0 de simetría del conjunto de capas a colaminar. Dado el sistema de referencia elegido (Figura 1) la función que representa el campo de velocidades del material de esta capa es sensiblemente más sencilla que la anterior. Al sustituir $\mathrm{y}=\mathrm{y}_{1}$ y $\mathrm{y}=\mathrm{y}_{2}$ por $\mathrm{y}=\mathrm{y}_{2}$ y $\mathrm{y}=0$, respectivamente, la función de corriente que corresponde al flujo de la capa inferior queda de la siguiente manera:

$$
\phi_{3}=Q_{2}\left\{\frac{y}{y_{2}}+C_{2} y\left(y-y_{2)}\right\}\right.
$$

en donde:

$\mathrm{Q}_{2}=$ Gasto volumétrico de la capa inferior.

$\mathrm{Y}_{2}=$ Frontera superior. (Interfase de los materiales).

$\mathrm{C}_{2}=$ Gradiente del perfil de velocidades lin eal para la capa in fe rior.

De esta forma, $C_{2}$ se asume, al igual que en el caso anterior, como una función cuadrática de $x$, de forma que:

$$
C_{2}(x)=a_{2} x^{2}
$$

y también $a_{2}$ será uno de los parámetros pseudo-independientes a optimar y representa exactamente lo mismo que $a_{1}$ para la capainferior.

Las componentes de velocidad en este caso se reducen a:

$$
U_{2}=\frac{\partial \phi_{3}}{\partial y}=Q_{2}\left\{\frac{1}{y_{2}}+2 C_{2}\left(y-\frac{y_{2}}{2}\right)\right\}
$$

$V_{2}=-\frac{\partial \phi_{3}}{\partial x}=-Q_{2} y\left[\frac{-y_{2}^{\prime}}{y_{2}^{2}}-C_{2}^{\prime}\left(y-y_{2}\right)+C_{2} y_{2}^{\prime}\right]$

En la zona previa a la de deformación plástica, el campo de velocidades está dado por la función de corriente.

$$
\phi_{4}=Q_{2}\left\{\frac{y}{e_{2 i}}\right\}
$$

El límite rígido-plástico para la capa inferior se obtiene de igual manera al plantear la continuidad entre las líneas de corriente $\left(\phi_{3}=\phi_{4}\right)$.

$$
y_{4}=y_{4}(x)=y_{2}+\frac{\left[\left(1 / e_{2 i}\right)-\left(1 / y_{2}\right)\right]}{C_{2}}
$$

Acorde a la consideración (5), si las velocidades de ambos materiales son iguales a la salida, se debe cumplir la siguiente relación:

$$
\frac{Q_{1}}{e_{1 f}}=\frac{Q_{2}}{e_{2 f}}
$$


con lo que $Q_{2}$ no resulta ser independiente, ya que depende exclusivamente de $Q_{1}$ y de la relación de espesores final.

\section{Cinemática de la deformación}

El tensor de rapidez de deformación para el caso en estudio es:

$$
\dot{\varepsilon}=\left[\begin{array}{ccc}
\dot{\varepsilon}_{x x} & \dot{\varepsilon}_{x y} & 0 \\
\dot{\varepsilon}_{y x} & \dot{\varepsilon}_{y y} & 0 \\
0 & 0 & 0
\end{array}\right]
$$

Los términos del ten sor se calculan a partir de:

$$
v_{i}=-\frac{\partial \phi}{\partial x_{i}}
$$

\section{Rapidez de deformación efectiva o equivalente}

Para el caso de deformación biaxial, la rapidez de deformación eficaz se expresa como:

$$
\left.\dot{\varepsilon}_{e q}=\dot{\bar{\varepsilon}}=\sqrt{\frac{2}{3}\left(\dot{\varepsilon}_{x x}^{2}\right.}+\dot{\varepsilon}_{x x}^{2}+2 \dot{\varepsilon}_{x y}^{2}\right)
$$

\section{Carga en los rodillos}

La fuerza de laminación expresada por unidad de ancho es estimada a partir de la siguiente ecuación:

$$
P=\frac{J \cdot R}{L \cdot U_{0}}
$$

$\mathrm{R}=$ Ra dio de los rodillos.

$\mathrm{L}=$ Longitud de la proyección del arco de contacto.

$\mathrm{U}_{0}=$ Velocidad lin eal de los rodillos.

\section{Endurecimiento}

Éste generalmente se puede expresar como:

$$
\sigma=K \varepsilon^{n}
$$

Donde $n$ se le conoce como coeficiente de endurecimiento por deformación y $\mathrm{K}$ es una constante de rigidez.

Una forma usual de tomar en cuenta el endurecimiento es a través de un esfuerzo promedio cuya forma general se expresa como:

$$
\bar{\sigma}=\frac{1}{\Delta \varepsilon} \int_{\varepsilon 0}^{\varepsilon f} \sigma d \varepsilon
$$

\section{Análisis de potencia}

Los términos de potencia considerados son:

1. Potencia interna consumida por deformación plástica $\left(W_{i}\right)$.

2. Potencia consumida por diferencia de velocidades tangenciales en los límites rígido-plásticos $\left(\mathrm{W}_{\mathrm{s}}\right)$.

3. Potencia consumida por fricción entre los rodillos y el material de las capas externas $\left(\mathrm{W}_{\mathrm{f}}\right)$.

4. Dado que se considera que los materiales se unen a la salida se debe to mar en cuenta la potencia disipada por fricción en la interfase de las capas que se colaminan $\left(\mathrm{W}_{\mathrm{fi}}\right)$.

donde:

Capa superior

$$
J=\text { Potencia total. }
$$




$$
\dot{W}_{i 1}=\sigma_{01} \int_{v} \dot{\varepsilon}_{e q} d V
$$

(Potencia interna consumida por deformación plástica).

$$
W_{s 1}=\frac{1}{\sqrt{3}} \sigma_{0_{1}} \int_{\Gamma_{3}} \Delta V \quad d S
$$

(Potencia consumida por diferencia de velocidad tangencial en la superficie de corte).

$$
W_{f}=\frac{m_{1}}{\sqrt{3}} \sigma_{0} \int_{\Gamma 1}|\Delta V| d S
$$

(Potencia consumida por fricción entre el rodillo y la capa superior).

$$
\begin{aligned}
& \text { Capa inferior } \\
& \dot{W}_{i 1}=\sigma_{02} \int_{v_{2}} \dot{\varepsilon}_{e q} d V
\end{aligned}
$$

(Potencia interna consumida por deformación plástica).

$$
W_{s 1}=\frac{1}{\sqrt{3}} \sigma_{02} \int_{\Gamma_{4}}|\Delta V| d S
$$

(Potencia consumida por diferencia de velocidad tangencial en la superficie de corte).

$$
\text { Interfase }
$$

$$
W_{f l}=\frac{m_{2}}{\sqrt{3}} \sigma_{02} \int_{\Gamma_{2}} \mid \Delta V \quad d S
$$

(Potencia consumida por fricción entre el ma te rial de la capa in fe rior y la capa su pe rior).

La diferencia de velocidades tangenciales en las superficies de corte se evalúan a partir de descomponer en sus componentes normal y tangencial a los campos de velocidades evaluados en los límites rígido-plásticos (superficies de corte). La figura 2 muestra la forma de descomponer las velocidades en los límites rígido-plásticos.

De lo anterior, resultan finalmente seis términos de potencia a evaluar. De forma que la potencia total J* queda expresada como:

$$
J^{*}=\dot{W}_{i 1}+\dot{W}_{i 2}+\dot{W}_{s 1}+\dot{W}_{s 1}+\dot{W}_{f}+\dot{W}_{f l}
$$

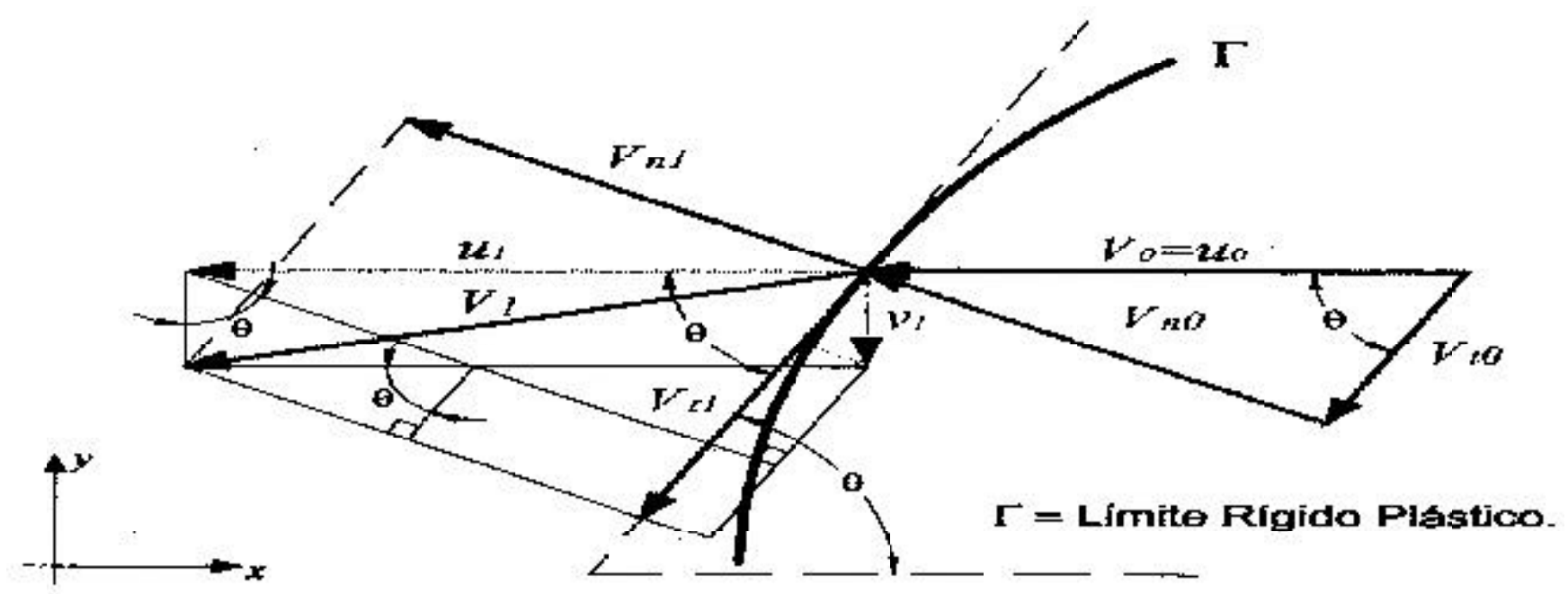

Figura 2 
La minimización de $J^{*}$, que finalmente queda en términos de $Q_{1}, b, a_{1}, a_{2}$ y $t_{\gamma}$ (los cuales resultan ser finalmente parámetros pseudo-independientes) es llevada a cabo mediante el método de búsqueda del poliedro flexible.

\section{Método del poliedro flexible}

Para el proceso de optimación se ha seleccionado un algoritmo rudimentario pero muy eficaz. Este esquema es pop u lar debido a lo robusto de su capacidad para encontrar mínimos en procesos de optimación de funciones cuyas variables no están acotadas 3 sin hacer uso de derivadas de la función.

En este caso, el algoritmo se ajusta perfectamente a la condición de la función objetivo $\left(0^{*}\right)$, ya que no resulta imposible calcular sus derivadas (porque J* está definida en términos de integrales), la forma de la función lo supone un proceso demasiado complejo o imposible.

De un análisis cualitativo del efecto de los parámetros sobre la configuración geométrica que adquiere el proceso, se desprenden las siguientes restricciones para los parámetros de optimación del caso en cuestión:

1. $\mathrm{b}>\mathrm{b}_{\min .}$ donde $\mathrm{b}_{\min .}=\frac{e_{2 i}-e_{2 f}}{x_{\text {cont. }}}$

y $x_{\text {cont. }}$ es la distancia desde el plano de salida del material hasta el punto donde el material de la capa superior hace contacto con los rodillos.

2. $a_{1}>0$.

3. $a_{2}>0$.
4. $\mathrm{Q}_{1}<0$.

5. $\left|Q_{t}\right|>\left|Q_{\min }\right|$, donde $\left|Q_{t}\right|=\left|Q_{1}\right|$ $+\left|Q_{2}\right| y\left|Q_{\min }\right|=\left|\omega \cdot R \cdot e_{m f}\right|$.

6. $e_{2 f}<e_{2 i}$.

7. $e_{1 f}<e_{1}$.

Estas restricciones pueden ser añadidas al modelo de optimación haciendo que el simplex se contraiga cuando se rebasan algunos de estos límites (cada restricción se puede visualizar como hiperplanos en un espacio de 5 dimensiones), esto se hace provocando que la función adquiera un valor muy grande, lo que induce la contracción del simplex, acotando de esta manera todos sus puntos dentro del lado permitido del hiperplano en cuestión.

Esta técnica es validada en la propuesta inicial del método desarrollado por Nelder y Mead, y en el caso en cuestión, evita entre otras cosas, que el valor de la función adquiera valores negativos, lo cual resulta ser físicamente inadmisible.

\section{Resultados}

Para efecto de validar el modelo desarrollado, se han corrido una serie de pruebas del algoritmo. A continuación se muestran algunos de los resultados que dan una idea del comportamiento gen eral del modelo (Tabla 1).

Los parámetros del proceso son:

$$
\begin{aligned}
& \mathrm{R}=200 \mathrm{~mm} \\
& \mathrm{~V}_{\mathrm{r}}=153.4 \mathrm{~mm} / \mathrm{s}
\end{aligned}
$$

3 Se mostrará más adelante la posibilidad y utilidad de restringir por medio de hiperplanos la región de búsqueda para el caso en cuestión. 
Se simula el proceso para valores de reducción de $10 \%, 20 \%, 30 \%, 40 \%, 50 \%$ y $60 \%$, variando en cada uno de ellos el factor de fricción entre el rodillo y el material de la capa superior en $0.2, \quad 0.4$ y 0.8 ; también considerando fricción sin deslizamiento entre los materiales de ambas capas que se colaminan en todos los casos.

La figura 3 muestra el efecto tanto de la reducción como del fac tor de fricción $n^{4}$ sobre la potencia total J*. El comportamiento es idéntico al que ocurre en el caso de laminación convencional. A bajos valores de reducción, el efecto de la fricción es menos no ta ble.

La figura 4 revela el efecto de la reducción y la fricción sobre la relación final de espesores de la capa inferior, donde $t_{f}$ es el espesor de dicha capa y $t_{f}$ es el espesor total. La relación de espesores inicial $\left(t_{2} / t\right)$ es 0.84 .
Las gráficas en las figuras 5 y 6 identifican el comportamiento del tamaño de las regiones de deformación plástica para cada una de las capas que se colaminan. Se puede apreciar la tendencia de dichas regiones a crecer conforme aumenta la reducción, (lo cual es de esperarse debido a que aumenta la severidad de la deformación); sin embargo, también se aprecia el poco efecto del factor de fricción sobre el comportamiento.

Para efectos de validar el modelo, se ha simulado el colaminado de un sandwich consistente en tres capas de aluminio de las mismas propiedades como una herramienta de comparación, por medio de un software de simulación especializado para procesos de conformado mecánico llamado Deform,

4 No confundir factor de fricción con coeficiente de fricción.

Tabla 1. Descripción de propiedades de los materiales a colaminar

\begin{tabular}{lcc}
\hline \multicolumn{1}{c}{ Parámetro } & Capa Superior & Capa Inferior \\
\hline Esfuerzo de cedencia & $70 \mathrm{MPa}$ & $270 \mathrm{MPa}$ \\
Espesor inicial & $1.2 \mathrm{~mm}$ & $6.3 \mathrm{~mm} *$ \\
Coeficiente de endurecimiento $(\mathrm{n})$ & 0.20 & $175 \mathrm{MPa}$ \\
Constante de rigidez $(\mathrm{K})$ & 0.25 & $500 \mathrm{MPa}$ \\
\hline
\end{tabular}

* El valor representa la mitad del espesor total de la capa intermedia

\section{Potencia}

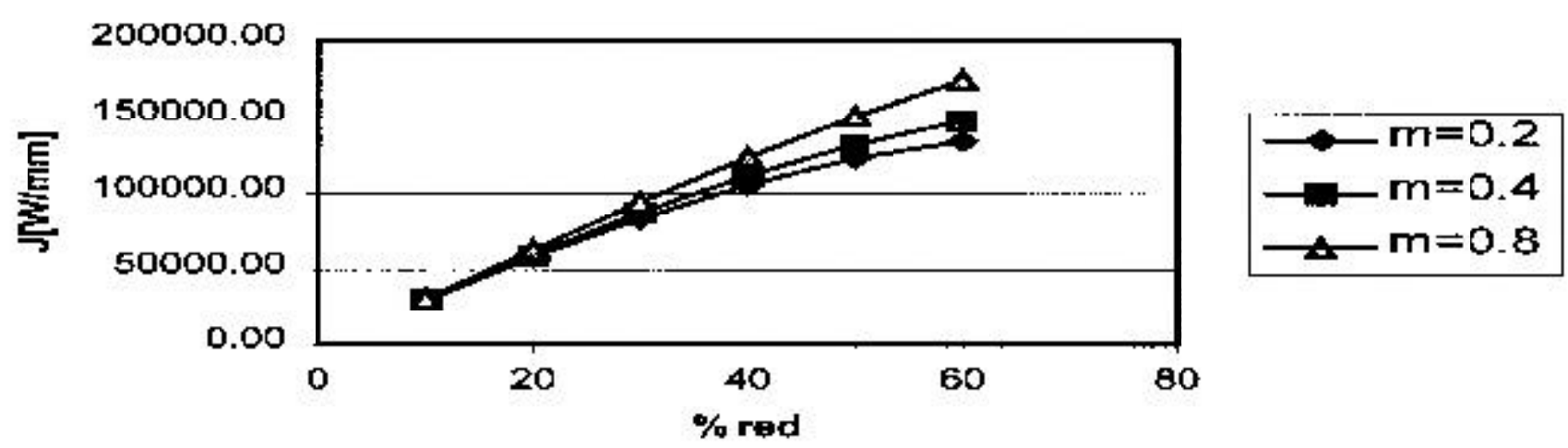

Figura 3 


\section{$\mathbf{t 2 f / t f}$}
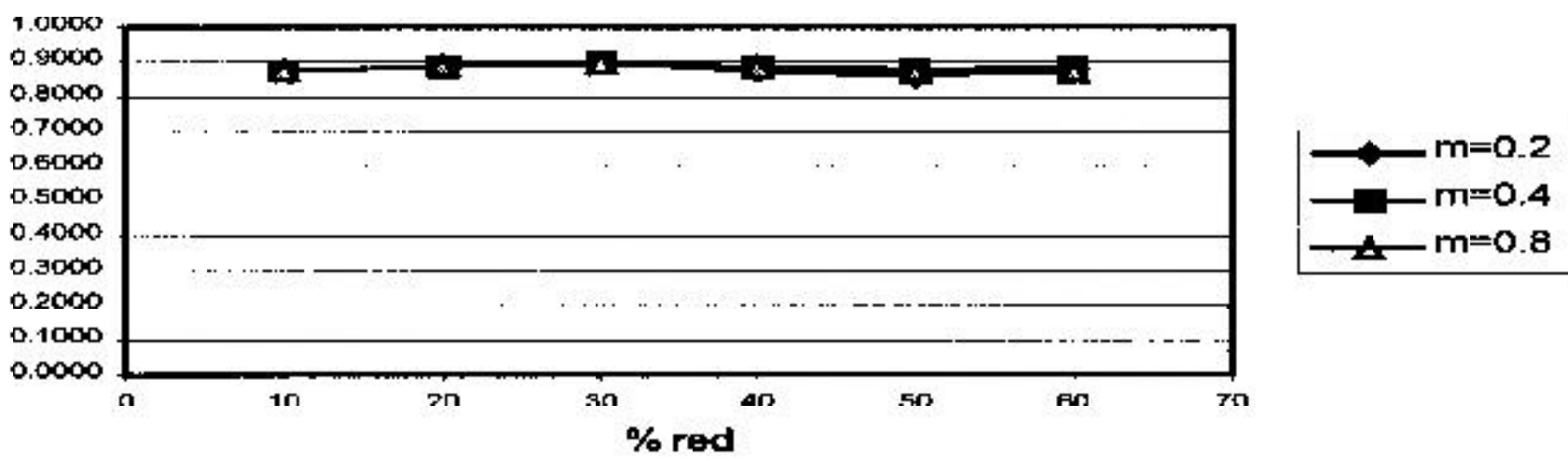

Figura 4

\section{Lit/L}
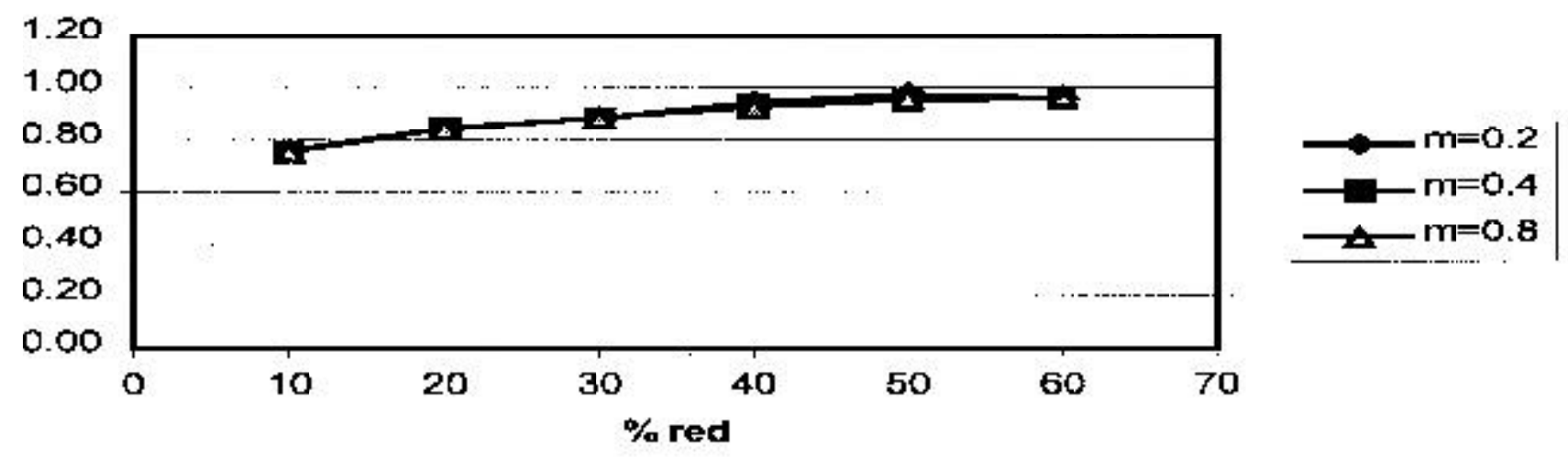

Figura 5

Li2/L

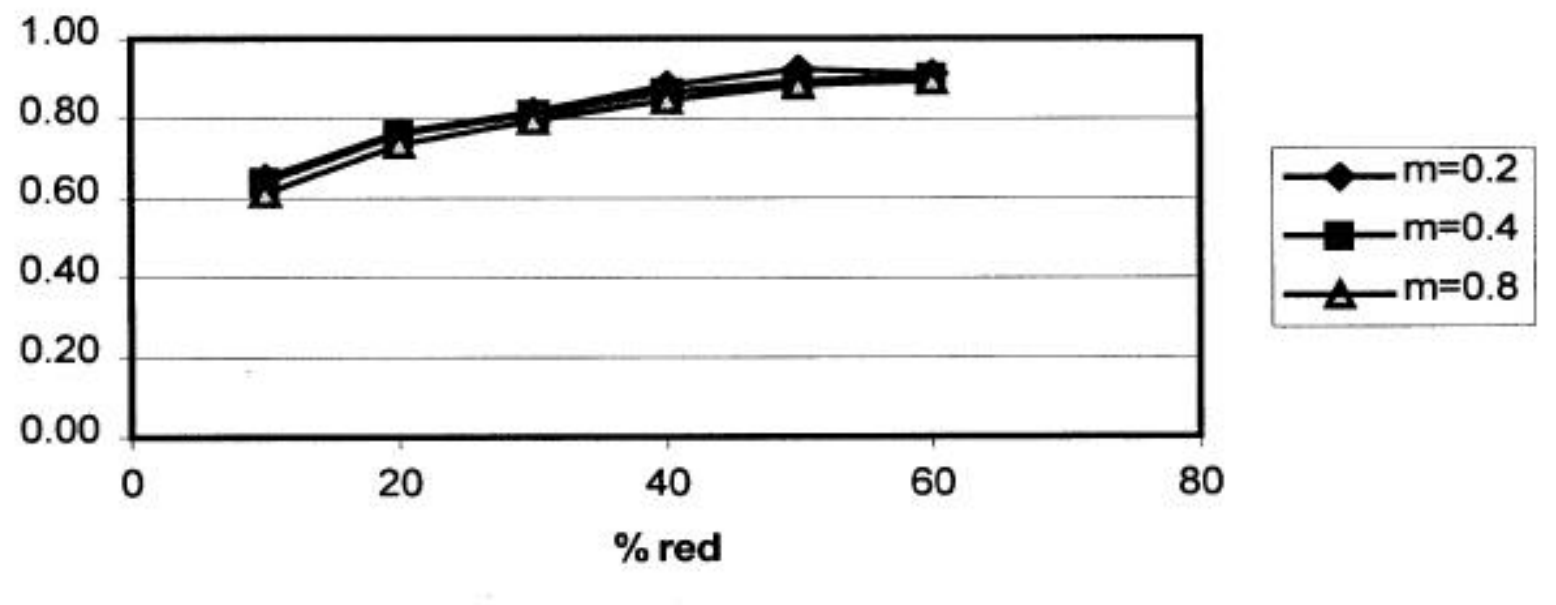

Figura 6 
Modelado y simulación de procesos de laminado conjunto por límite supe rior

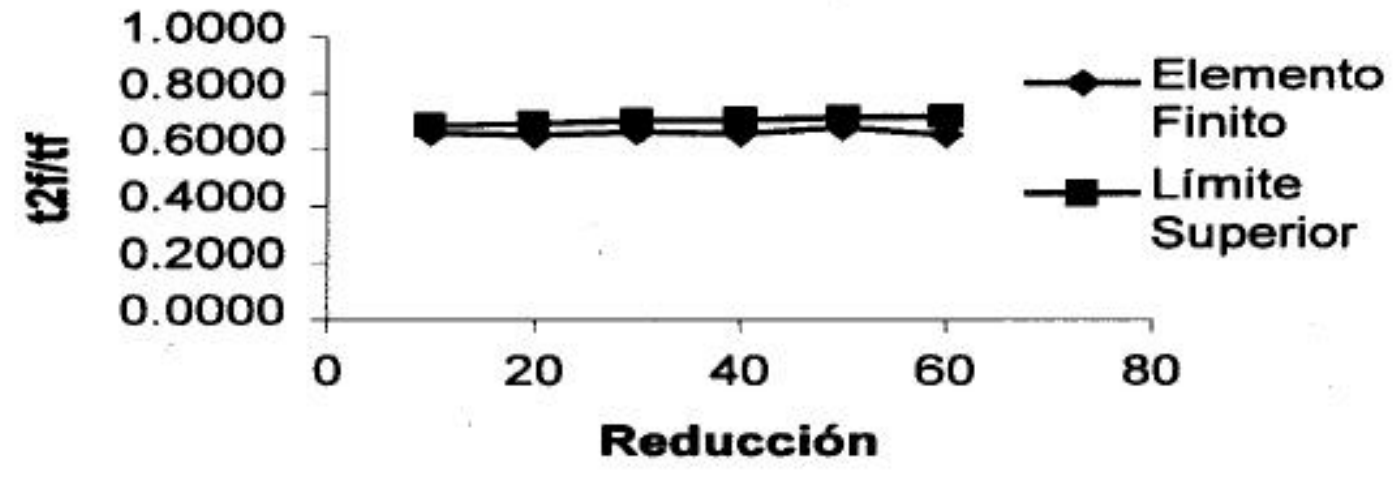

Figura 7

asimismo se compararon los resultados relativos a la deformación relativa entre las capas al final del proceso. La figura 7 muestra la comparativa entre las dos soluciones.

Aparentemente, la solución por elemento finito, que sí considera el equilibrio de momentum durante el proceso (equilibrio estático) predice una mayor deformación en la capa inferior de aquella predicha por la solución en Limite Superior. La diferencia más grande entre ambas soluciones es del orden del $4.82 \%$.

Finalmente, cabe mencionar que el tiempo de optimación oscila entre 20 y 50 minutos, en función del sim plex, el cual se de fine en un inicio del proceso. Esto hace que la duración de una corrida completa para una cierta combinación de espesores de capas y materiales se extienda hasta una jornada de trabajo.

\section{Análisis de pseudo-parámetros}

En el modelo desarrollado son cinco los parámetros que se optimizan y dan forma a la mejor aproximación del campo de velocidades de ambas capas de materiales.
$a_{1}$ - Gradiente de distribución de velocidad horizontal capa su pe rior.
$a_{2}$ - Gradiente de distribución de velocidad horizontal capa in fe rior.
b - Amplitud de la parábola que de scribe la forma de la interfase entre capas.
$\mathrm{Q}_{1^{-}}$Gasto volumétrico por unidad de ancho de la capa su pe rior.
$t_{\gamma^{-}}$Mitad del espesor final de la capa inferior.

Se ha estudiado la evolución de los pseudo-parámetros durante diversas condiciones de laminación. El comportamiento en todos los casos es básicamente el mismo, ya que $\mathrm{a}_{1}$ y $\mathrm{a}_{2}$ son los parámetros que más tardan en estabilizarse. Además, dichos parámetros presentan la peculiaridad de que pequeños cambios en sus valores provocan variaciones considerables del valor de la función objetivo con relación a la tolerancia que sive como criterio para detener el proceso de optimación. Por lo anterior, en repetidas ocasiones es necesario controlar el valor de dicha tolerancia para evitar caer en ciclos infinitos du rante la optimación.

\section{Discusión}


H.A. Quiroz-González1, A. Ortiz-Prado², Y. Houbaert-Irmen

Es evidente que la fricción juega un papel poco importante sobre la deformación relativa de los materiales colaminados.

Contrario a lo que en un principio se esperaba, para el caso analizado, la relación de espesores se mantiene casi constante a pesar de variar tanto la reducción como el factor de fricción entre el rodillo y el material de la capa superior.

Para el caso de la potencia disipada por fricción entre las capas que se colaminan, se ha asumido el modelo de fricción constante y un fac tor de fricción igual a uno, con lo que se supone fricción sin deslizamiento, que en el caso de superficie preparadas previamente, el comportamiento se debe acercar en buena medida a dicha suposición.

De la figura 6 se puede apreciar el hecho de que la variación en la extensión de la región plástica es más notoria en el caso de la capa su pe rior, esto obedece al hecho de que es esta la capa más rígida, por lo tanto, menos susceptible a deformarse antes que la capa inferior que es menos rígida. Sin embargo, conforme se aumenta la reducción la capa inferior se ve forzada a una mayor deformación, ya que al aumentar la reducción crece el esfuerzo necesario para deformar la capa supe rior (que es aproximadamente 10 veces más delgada que la inferior), esto da como resultado la afectación del balance entre potencias internas de deformación a favor de una menor deformación en la capa superior, empleando el criterio (LS).

\section{Conclusiones}

Con el modelo desarrollado, se cuenta con una herramienta útil en la predicción de la cinemática del proceso. El Teorema de Límite
Superior asegura que el mínimo de la función de potencia total conduce a un campo de velocidades que será muy próximo al verdadero. Cualquier inferencia hecha respecto al campo de esfuerzos asociados al campo de deformaciones debe ser tratada con mucha precaución.

La fricción y la reducción juegan un papel poco significativo en deformación relativa de las capas que se colaminan.

En un inicio se pensó que el comportamiento de la solución no sería lineal respecto al endurecimiento que presentan los materiales. Lo anterior implicaba la hipótesis de que la solución se vería afectada en función del endurecimiento y la deformación individuales de cada uno de los materiales de las capas que se colaminan. Sin embargo, la variación entre una solución que considere endurecimiento y otra que no, resulta ser insignificante.

El modelo de optimación utilizado se comporta adecuadamente la mayoría de las veces. No obstante, se pudo observar que hay casos en donde dos mínimos y la solución oscila entre ambos posibles valores en función del simplex inicial. Pese a lo anterior, es fácil ubicar aquellos casos en lo que ocurre tal inconveniente, para ello, basta con analizar el comportamiento de las curvas respectivas y ubicar aquellos puntos del simplex final que por sí resultan ser físicamente inadmisibles y que provocan una variación notable en el comportamiento de las curvas como las mostradas en las figuras $3,4,5$ y 6 , y entonces repetir la simulación para aquellas condiciones variando la configuración del simplex inicial hasta lograr una solución coherente.

El análisis del comportamiento de los pseudo-parámetros durante el proceso de 
optimación sugiere que tanto $a_{1}$ como $a_{2}$ son demasiado inestables, lo cual puede ser el resultado de la pequeña aportación del perfil linealmente distribuido, por lo que se manifiesta en valores pequeños para dichos parámetros (de un orden en ocasiones menor a $\left.10^{-9}\right)$, donde el principal inconveniente es el efecto de la precisión de la máquina al operar con valores tan pequeños. Lo anterior se puede mejorar al plantear una función más adecuada para el gradiente que representan tanto $a_{1}$ como $a_{2}$.

\section{Referencias}

Avitzur-Betzalel. (1980). M etal Forming. The Application of Limit Analysis. 1st Edition. Marcel Dekker Inc. USA, ISBN 0-8247-6847-7.

Nelder J.A. y Mead R. A Simplex Method for Function Minimization. Journal of Computer Science, Vol. 3, No. 10, pp. 308-313.

Yeong-Maw H., Hung-Hsiou H., Hung-Jen L. (1995). Analysis of Sandwich Sheet Rolling by Stream Func tion Metho d. International Journal of Mechanical Science, Vol. 37, No. 3, pp 297, 315. Elsevier Science.

\section{Bibliografía sugerida}

Arnold R.R., Whitton P.W. (1959). Stress and Deformations Studies for Sand wich Rolling Hard Metals. Proc. Instn. Mech. Engrs, 173 (8), pp. 241-256.

Hosford W.F. y Caddell R.M. (1993). Metal Forming. Mechanics and Metallurgy. $2^{\text {nd }}$ Edition. Prentice Hall.USA, ISBN 013 5885264.

Kobayashi-Shiro, Soo-lk 0. Altan T.

(1989). M etal Forming \& The Finit Element
Method. $1^{\text {st }}$ Edition.Oxford University Press, USAISBN 0195044029.

Quan Z. (1987). Solid Phase Roll Cladding of Bimetallic Strip by Cross Shear Cold Rolling. Advanced Technology of Plasticity, Vol. II, p. 789.

Rardin R.R. (1998). O ptimization in 0 perations Research. $1^{\text {st }}$ Edition. Prentice-Hall, Inc. USA, ISBN 0023984155.

Richelsen A.B. (1997). Elastic-Plastic Analysis of the Stress and Strain Distribution in Asymmetric Rolling. International Journal of M echan ical Sciences, Vol. 39, No. 11, pp. 1199-1211.

Rowe G.W., Sturgess C.E.N., Hartley P., Pillinger I. (1991). Finit Element Plas ticity and Metal Forming Analysis. $1^{\text {st }}$ Edition. Cambridge University Press. Great Britain, Cambridge, ISBN 0521383625.

Shi-Hoon C. (1997). Tensile Deformation Behavior of Stainless Steel Clad Aluminum Bilayer Sheet. Materials Science and Engi neering, A222, pp. 158-165.

Talbert S.H. y Avitzur-Betzalel. (1996). Elementary Mechanics of Plastic Flow In Metal Forming. 1st Edition. John Wiley and Sons. England, ISBN 0471960039.

Tzou G.Y. (2001). Theorical Study on the Cold Sandwich Sheet Rolling Considering Coulomb Friction. Journal of $M$ aterials Processing, No. 114, pp. 41-50.

Wusato wsky-Zigmunt. (1969). Fundamentals of Rolling. $1^{\text {st }}$ Edition. Pergamon Press. Poland.

Yeong-Maw H., Hung-Hsiou H., Yuh-Lin $\mathrm{H}$. (2000). Analytical and Experimental Study on Bonding Behavior at the Roll Gap during Complex Rolling of Sand wich Sheets. International Journal of Mechanical Sciences, No. 42, pp. 2417-2437. 
Yong J., Dashu P., Dong L.L. (2000). Analysis of Clad Sheet Bonding by Cold Rolling. Journal of Materials Processing Technology, pp. 32-37.

\section{Semblanza de los autores}

H éctor Q uiroz-G onzález. Es ingeniero mecánico egresado de la Facultad de Ingeniería de la UNAM, cursó los estudios de posgrado en ciencia e ingeniería de materiales en el Instituto de Investigaciones de Materiales de la misma Universidad. Actualmente labora para el Centro de Ingeniería Avanzada en Turbomáquinas en la ciudad de Querétaro, en el Área de Diseño e Ingeniería Estructural. Sus campo de interés e investigación com prende lo relacionado con metalurgia, modelado de procesos de deformación plástica en metales y análisis numérico aplicado al diseño de estructuras para motores aeroderivados.

Armando 0 rtiz-Prado. Es ingeniero mecánico electricista y maestro en ingeniería mecánica por la Facultad de Ingeniería, UNAM. En 1989, obtuvo la distinción Universidad Nacional para Jóvenes Académicos en el área de docencia en ciencias exactas. Ha dirigido más de 70 tesis entre licenciatura y posgrado, así como más de 50 estudios y proyectos para el sector industrial en el área de manufactura, materiales, y particularmente, en anális de falla. Tiene más de 40 publicaciones relacionadas con el área de su especialización en fo ros nacionales e internacionales. Actualmente es profesor y coordinador de la Unidad de Investigación y Asistencia Técnica en Materiales.

Yvan H oubaert-Irmen. Graduado como ingeniero metalúrgico en la Universidad de Gante, Bélgica en 1969, obtuvo su doctorado en ingeniería mecánica en la Universidad de Hannover, Alemania en 1973. De 1975 a 1980 fungió como profesor en la Facultad de Ingeniería de la UNAM y fue jefe del Departamento de Ingeniería Mecánica de 1978 hasta su retorno a Bélgica, en donde fue reclutado en octubre de 1980 para colaborar como académico de tiempo completo en el área de Metalurgia. Desde 1997 a la fecha, labora como jefe del Departamento de Metalurgia y Ciencia de Materiales y como director del Laboratorio de Siderurgia en la Universidad de Gante. A la fecha tiene publicados más de 100 artículos en revistas y en los foros más destacados de su área. 